NASA-TM- 111559

\title{
Small uitranign vacuum compatible hyperthermal oxygen atom generator
}

\author{
R. A. Outlaw \\ NASA Langley Research Center, Hampton, Virginia 23681-000I \\ Mark R. Davidson \\ DACO Technologies, Inc., Florahome, Florida 32140
}

(Received 1 March 1993; accepted 5 February 1994)

\begin{abstract}
The development of a compact, ultrahigh vacuum (UHV) compatible instrument for generating a flux of pure hyperthermal oxygen atoms for NASA applications has been achieved. The instrument combines the mechanisms of $\mathrm{O}_{2}$ dissociation and transport through a hot $\mathrm{Ag}$ membrane to provide a continuous source of $\mathrm{O}$ atoms to a vacuum interface where they are subsequently emitted into the vacuum space by electron-stimulated desorption (ESD). A flux of neutral $\mathrm{O}$ atoms $4.5 \times 10^{1.3}$ $\mathrm{cm}^{-2} \mathrm{~s}^{-1}\left({ }^{3} \mathrm{P}\right)$ with a mean ion kinetic energy of approximately $5 \mathrm{eV}$ and a full width at half maximum of $4 \mathrm{eV}$ was detected at a quadrupole mass spectrometer located $10 \mathrm{~cm}$ away. The geometry of the instrument is such that it is mounted on a 7-cm flange and can be tailored in length and orientation to fit most UHV systems. The data presented here are for ESD-controlled conditions where increases in the flux are strictly linear with electron bombardment current. Calculation shows that transport-controlled conditions can be achieved at temperatures as low as $350{ }^{\circ} \mathrm{C}$ with membrane thicknesses on the order of $10 \mu \mathrm{m}$.
\end{abstract}

\section{INTRODUCTION}

The composition of the atmosphere within the orbital envelope $(200-1000 \mathrm{~km})$ combined with the orbital velocity $\left(\sim 8 \mathrm{~km} \mathrm{~s}^{-1}\right)$ results in a flux of hyperthermal atomic oxygen $\left(E \sim 5 \mathrm{eV}, \nu \sim 10^{15} \mathrm{~cm}^{-2} \mathrm{~s}^{-1}\right)$ impinging on spacecraft surfaces. The extreme reactivity of atomic oxygen leads to numerous chemical unions with other species to form, for example, $\mathrm{CO}, \mathrm{CO}_{2}, \mathrm{H}_{2} \mathrm{O}, \mathrm{O}_{2}, \mathrm{SO}_{2}$, and $\mathrm{NO}_{2}$. Further, the high chemical reactivity of this $\mathrm{O}$ atom flux has caused substantial degradation of organic materials on board the Space Shuttle and the Long Duration Exposure Facility (LDEF) and suggests that materials on the proposed space station the composites used in large space structures, exterior coatings on the optics of the Hubble space telescope, the UV telescopes, and future laser communications systems may have substantially reduced lifetimes. It is, therefore, essential to study the reactivity of these materials to atomic oxygen in ground based laboratories. ${ }^{1-3}$ In order to conduct such laboratory experiments, an atomic oxygen beam generator is required that can accurately simulate the flux and energy (within the appropriate vacuum environment) vehicle's experience in orbit. In addition to oxygen atom reactions with spacecraft materials, such a beam system would also be of importance to NASA in the calibration of mass spectrometers and other detection systems that would be used for mapping the density of the gas constituents within the orbital envelope. Calibration is essential to make accurate measurements of the representative gas environment within the orbital envelope. A pure, well-behaved $O$ beam would also be useful for producing an ordered oxide layer for the growth of compound semiconductors and superconductors. Methods of thin film growth, such as molecular beam epitaxy (MBE), could utilize a directed $\mathrm{O}$ beam to grow desired oxide layers without the residual contaminating effects of backfilled $\mathrm{O}_{2}$ or the limitations of dissociative adsorption. Other areas of interest, such as fundamental surface science and chemical ki- netics, are obvious. There are several systems that are presently available for the above applications, but they are, in general, quite large, expensive, not UHV compatible, and require the samples to be brought to its location. ${ }^{4-6}$ The instrument presented here is small (fits on a $7-\mathrm{cm}$ flange), simple, and designed for UHV applications.

\section{DESIGN CONCEPTS}

The hyperthermal oxygen atom generator (HOAG) employs the use of two different mechanisms. The first is the unusually high permeability of oxygen through $\mathrm{Ag}$ and its alloys which occurs by the sequential adsorption of $\mathrm{O}_{2}$, surface dissociation into atoms, dissolution into the bulk, and diffusion of $\mathrm{O}$ atoms through a thin membrane where the atoms emerge at the vacuum interface and enter into atomically bound states at the surface $\left(T<550^{\circ} \mathrm{C}\right)$. At higher temperatures $\left(T>550^{\circ} \mathrm{C}\right)$, the $\mathrm{O}$ atoms which arrive at the vacuum interface have sufficient thermal energy for surface diffusion which ultimately results in recombination and desorption of $\mathrm{O}_{2}$ molecules. The second mechanism is the utilization of electron-stimulated desorption (ESD). By using an incident flux of low energy electrons $(E<3 \mathrm{kV})$, the bound atoms are excited to antibonding states and desorb as $\mathrm{O}$ neutrals with hyperthermal kinetic energies. Figure 1 shows the combination of these two processes schematically. Usually, ESD is studied on dosed surfaces where the emission is a function of the decay in surface coverage. Eventually, the coverage becomes so low that redosing is required to continue the study. In the case presented here, a continuous source of molecular oxygen is provided (upstream), usually $10-1000$ Torr, to resupply the UHV interface (downstream) by permeation through the $\mathrm{Ag}$ membrane. This, of course, means that the $\mathrm{Ag}$ must be operated at a temperature high enough to ensure a sufficient oxygen permeability, but yet low enough that the atomic adsorbed state 


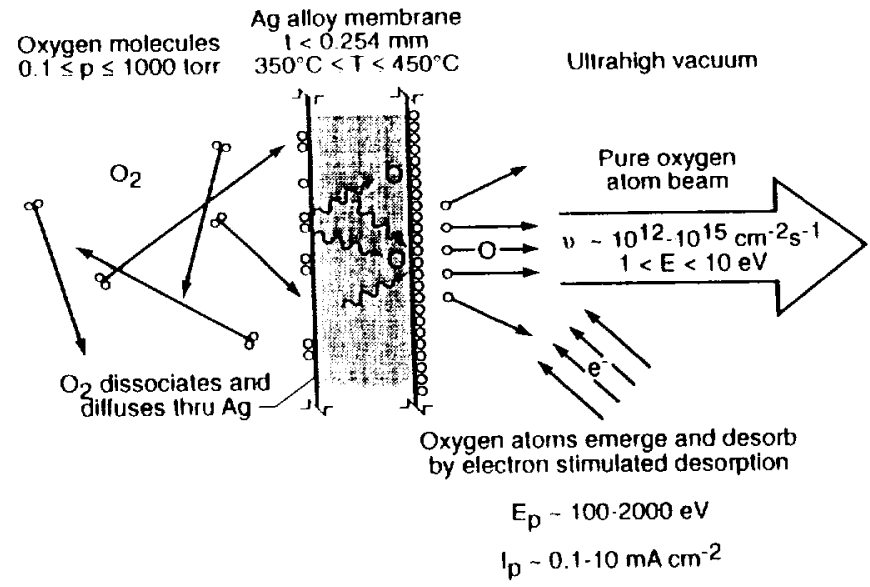

FIG. I. Schematic representation of the combined mechanisms of oxygen transport through a $\mathrm{Ag}$ or $\mathrm{Ag}$ alloy membrane followed by emission of hyperthermal $O$ neutrals by ESD.

is stable, and molecular oxygen is not formed. The surface coverage of oxygen atoms downstream at the UHV interface is given by

$$
-\sigma_{0} \frac{d \theta}{d t}=J_{\mathrm{O} / \mathrm{Ag}}-J_{\mathrm{O} / \mathrm{ESD}},
$$

where $\theta$ is the surface coverage, $J_{\mathrm{O} / \mathrm{Ag}}$ is the oxygen atom flux through the $\mathrm{Ag}, J_{\mathrm{O} / \mathrm{ESD}}$ is the electron stimulated desorption of oxygen, and $\sigma_{0}$ is the monolayer surface concentration $\left(\sim 2.4 \times 10^{15} \mathrm{~cm}^{-2}\right)$. The limiting mechanism is determined primarily by membrane temperature, thickness, and upstream pressure for the transport and by electron bombardment flux and energy for ESD.

The original proof of concept of these combined mechanisms was accomplished by charging a Ag wire with 100 Torr of oxygen at $500^{\circ} \mathrm{C}$ for $1 \mathrm{~h}$ to provide a high concentration of oxygen in solid solution. ${ }^{7}$ Then, after evacuation to the UHV region, the wire was heated while simultaneously bombarding the surface with $100-\mathrm{eV}$ electrons at a current of $0.5 \mathrm{~mA}$ in direct line of sight to the ion source of a quadrupole mass spectrometer (QMS). A very clear and unambiguous atomic oxygen peak was observed and found to behave predictably and characteristically on the relevant transport and ESD parameters.

\section{PROTOTYPE HOAG}

\section{A. Instrument geometry}

Various scientific and technological studies connected with the development of a device which employs the combined mechanisms shown in Fig. 1 have been previously reported. $^{8}$ Figure 2 shows a sketch of the HOAG (without cover plate) mounted on an 8-pin, 7-cm diameter conflat flange. The overall length can be constructed to fit any size $(>10 \mathrm{~cm})$ or desired curvature. The intermediate flange is for simple replacement of the membrane assembly shown in the figure inset (without heater). The heater is two concentric layers of $0.025-\mathrm{cm} \mathrm{NiCr}$ wire coils mounted on a macor mandrel which can provide sufficient heat to adjust the mem-

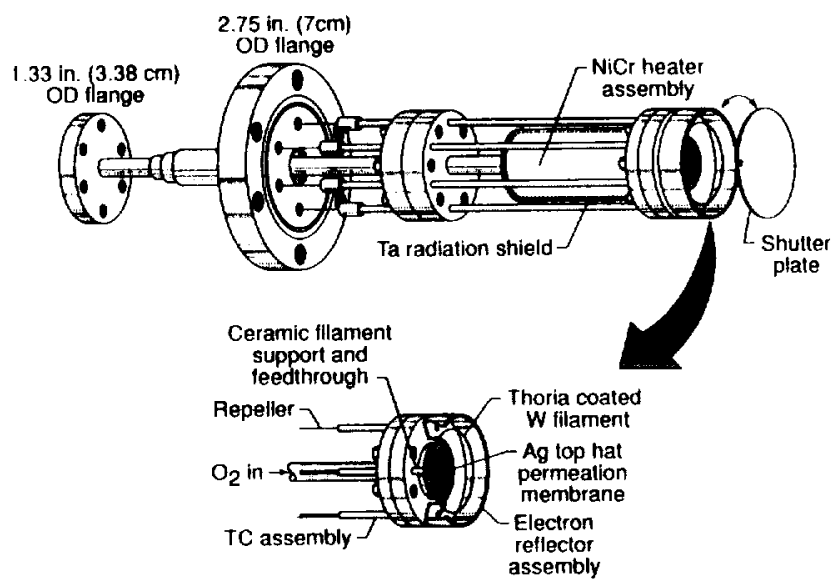

Fig. 2. Sketch of the HOAG assembly with detailed inset of the tophat membrane, circular cathode, and reflector.

brane to temperatures in excess of $800^{\circ} \mathrm{C}$ (anything above $550^{\circ} \mathrm{C}$ is used to clean the membrane. The circular thoria coated $\mathrm{W}$ cathode is $0.025 \mathrm{~cm}$ in diameter and located in a plane that is $0.1 \mathrm{~cm}$ higher than the surface of the $\mathrm{Ag}$ or $\mathrm{Ag}$ alloy top-hat membrane. The cathode is concentric with a computer designed electron reflector to ensure a uniform electron bombardment over the membrane which will generate a uniform atomic oxygen flux. The inset also shows a shutter plate to minimize membrane or target contamination prior to procedural cleanup and also to provide an instant turn-on and turn-off capability. The membrane is also insulated from the flange to allow a bias voltage for optimun electron and ion optics. The atomic-oxygen experiments were conducted in a UHV ion and turbo-pumped system with an ultimate pressure less than $1 \times 10^{-10}$ Torr. The HOAG was positioned axially $10 \mathrm{~cm}$ away from an EAl Quad 250 QMS equipped with an axial beam ionizer. The' instrument was operated in the pulse counting or analog modes and was used for the direct measurements of the neutral and ion flux levels. Details of this system have been previously published. ${ }^{8}$ The ionizer was operated using a beam of $70-\mathrm{eV}$ electrons. Typically, the ions formed are accelerated into the quadrupole region using a negative potential in the range of 3-10 V. Lower-energy ions have a higher probability of following an unstable path, so more of them leave the quadrupole field and are not detected. The cutoff acceleration potential was determined to be $2 \mathrm{~V}$ by observing the threshold for thermal ions as the acceleration potential was reduced. Therefore, by reducing the accelerating potential to $0 \mathrm{~V}$, higher kinetic energy ESD neutrals $(>2 \mathrm{eV})$ are detected while background neutrals are not. The only back. ground signal observed in this mode consists of very small peaks at 12 and $16 \mathrm{amu}$, which are due to the energetic: fragmentation of $\mathrm{CO}$. This interference can be removed by operating the ionizer electron energy in the appearance potential (AP) mode. The ionizer was operated below the AP for $\mathrm{CO}$ but above the AP for $\mathrm{O}$. An ionizer-electron acceleration potential of $22 \mathrm{~V}$ was found to be low enough to elimi- 


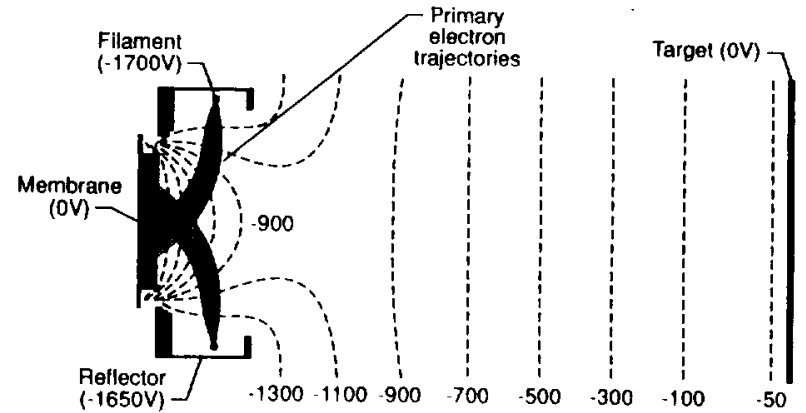

Fig. 3. Simion computer simulation of the electron trajectories for the cathode/reflector assembly shown in Fig. 2. Note the uniform flux over the Ag emission plane. Equipotential lines are also presented.

nate 12- and 16-amu background peaks in the QMS. At $22 \mathrm{~V}$ the background signal was reduced to less than 0.25 counts.

\section{B. Computer-aided design}

Figure 3 shows the results of the computer-aided design of the electron trajectories using the reflector/cathode arrangement shown in Fig. 2. When the membrane is operated at ground and the cathode is operated at $-1700 \mathrm{~V}$, the reflector is operated at $+50 \mathrm{~V}$ with respect to the cathode which gives the uniform bombardment flux as shown. The arrangement of the lens and filament creates a cylindrical "saddle" field as shown by the dashed equipotential surfaces. While the saddle field does not accelerate electrons near the membrane toward the membrane, it accelerates positive $O$ ions toward the target. As these ions leave the area near the membrane, they must climb back out of the saddle field. Positive ions that are emitted normal to the membrane surface and on the axis do reach the target since both the target and the membrane are at the same potential. As shown in Fig. 4(a), the ions emitted in parallel paths near the membrane centerline (normal to the surface) have sufficient energy to escape the reflector potential field. However, if a bias voltage of $-80 \mathrm{~V}$ is applied to the membrane, any ions with energies of less than $80 \mathrm{eV}$ will not have sufficient energy to reach the target. The highest kinetic energy $O$ ions for the system are $10 \mathrm{eV}$, so all ion emission will be effectively trapped [see Fig. 4(b)]. A separate electrode in front of the emission plane to capture all the ions is also an option, but it is desirable to minimize any scattering or recombination surfaces that may alter the emission flux or purity. Further, this bias does not affect the ESD of $\mathrm{O}$ neutrals in any significant way.

\section{Performance characteristics}

The HOAG spectra as detected by the QMS with the ionizer in the appearance potential mode and the extractor voltage at $0 \mathrm{~V}$ is shown in Fig. 5. The QMS signal, due to background gases, cuts off at extractor potentials less than 2 $\mathrm{V}$ which indicates that ions with energies less than $2 \mathrm{eV}$ have a negligible probability for transmission through the quadrupole mass filter. At an extractor potential of $0 \mathrm{~V}$ the ESD neutrals are detected, but background signals are not. This indicates that ESD neutrals of $2 \mathrm{eV}$ or greater are detected.

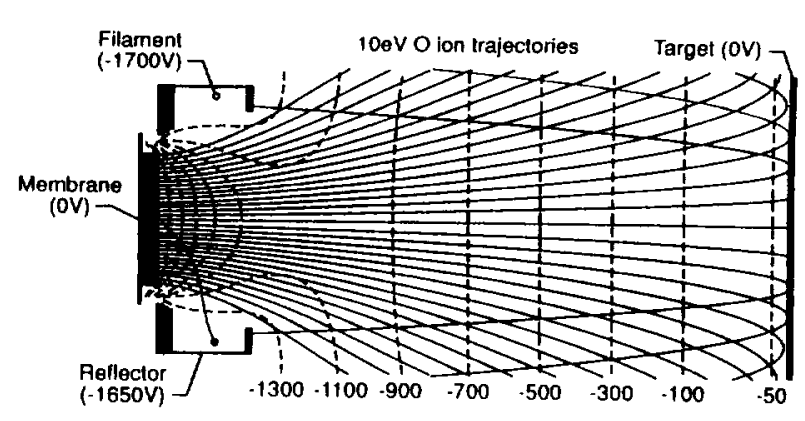

(a)

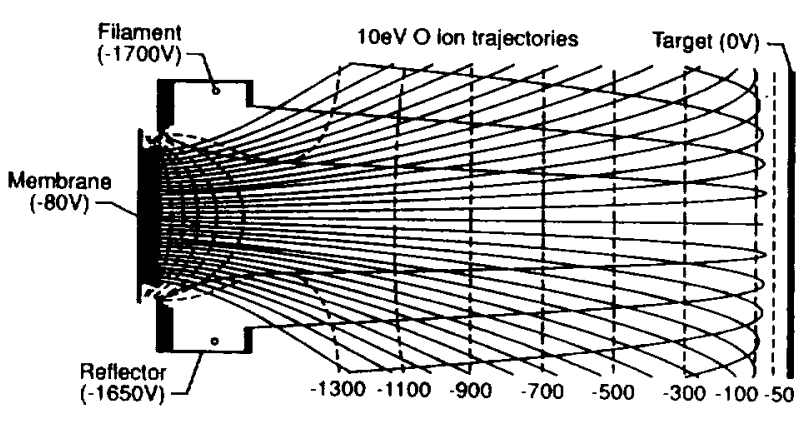

(b)

FiG. 4. Simion computer simulation of the ion trajectories for a sample to target distance of $9 \mathrm{~cm}$. Some ions strike the target. (b) Applying a bias of $-80 \mathrm{~V}$ returns all ions to the emission plane, eliminating the need for a separate grid. Equipotential lines are also presented.

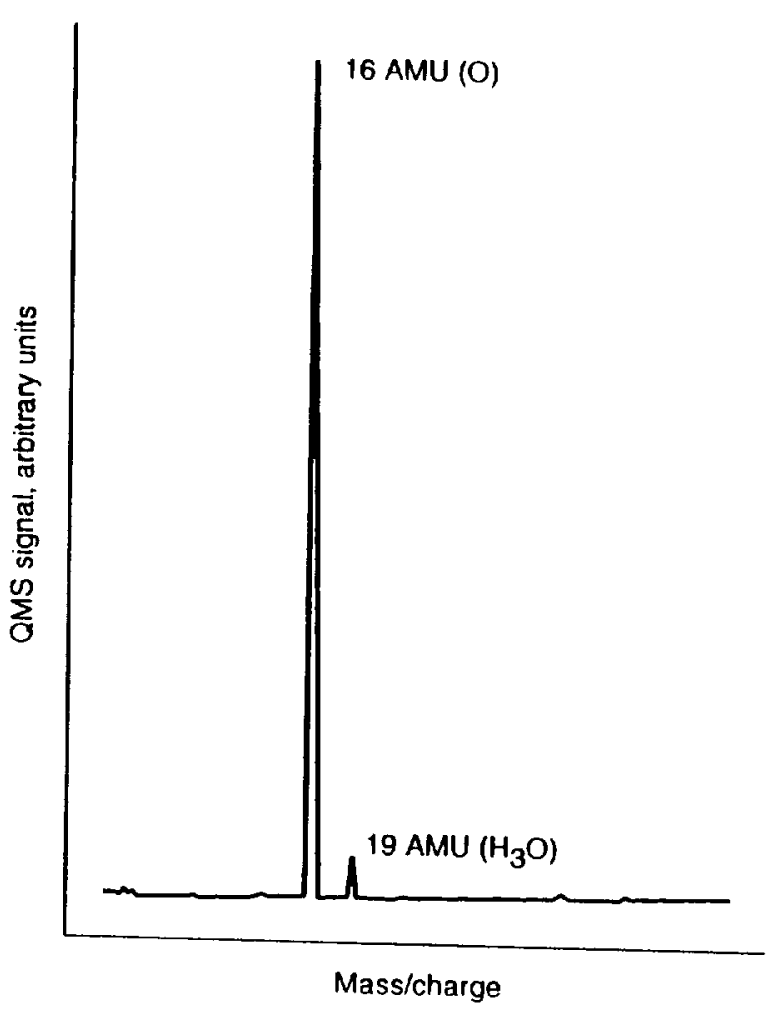

FIG. 5. Atomic oxygen signal for the instrument as detected by the QMS in the appearance potential mode. The bias on the extractor plate of the ionizer is adjusted to restrict thermals. 
Clearly, the predominant peak is atomic oxygen. The continuously diminishing peak at $m / e=19$, which is most likely $\mathrm{H}_{3} \mathrm{O}$ from the residual adsorbed $\mathrm{H}_{2} \mathrm{O}$, will ultimately disappear after sufficient clean up. The ionizer efficiency is the ratio of the oxygen atom ion flux exiting the ionizer $\left(J_{\text {out }}\right)$ to the oxygen atom flux entering the ionizer $\left(J_{\text {in }}\right)$ which for this QMS was determined from

$$
\frac{J_{\text {out }}}{J_{\text {in }}}=\frac{S k_{B} T}{A e v_{\text {in }}}
$$

where $S$ is the QMS sensitivity, $1 \times 10^{-4}$ A/Torr $\left(\mathrm{O}_{2}\right)$ at 0.2 $\mathrm{mA}$ emission current; $k_{B}$ is Boltzmann's constant, $1.38 \times 10^{-23} \mathrm{~J} \mathrm{~mol}^{-1} \mathrm{deg}^{-1} ; T$ is the gas temperature, $\mathrm{K} ; A$ is the area of the aperture, $0.508 \mathrm{~cm}^{2}$; $e$ is the electronic charge, $1.6 \times 10^{-19} \mathrm{C}$; and $v_{\text {in }}$ is the velocity of the entering particles, $7.7 \times 10^{15} \mathrm{~cm} \mathrm{~s}^{-1}$. Equation (2) yields an efficiency of $6.4 \times 10^{-8}$ ions/atom for an emission current of $0.2 \mathrm{~mA} .{ }^{9}$ The emitted ESD ions apparently experience a high probability of neutralization, but we are uncertain as to the reason for this. The variation in atomic oxygen flux as a function of incident electron flux is shown in Fig. 6. The QMS data taken in Fig. $6(\mathrm{a})$ is by pulse counting at an electron energy of $1 \mathrm{keV}$ and a membrane temperature of $550^{\circ} \mathrm{C}$. The linearity is consistent with normal ESD behavior and indicates that the HOAG is ESD limited. Figure 6(b) shows analog data (pulse counting was not used because of a saturated multiplier) giving the highest flux achieved so far, $4.5 \times 10^{13} \mathrm{~cm}^{-2} \mathrm{~s}^{-1}\left(I_{e}=10 \mathrm{~mA}\right)$ at a distance of $10 \mathrm{~cm}$ from the emission plane. The primary limitation is controlling the temperature of the membrane at $28 \mathrm{~W}$ input of power $(10 \mathrm{~mA}$ at $2.8 \mathrm{kV})$. The new design of the instrument employing a thick walled $\mathrm{Ag}$ oxygen-supply tube instead of stainless steel permits control of the membrane temperature.

The dependence on the source of molecular oxygen upstream is shown in Fig. 7. A steady state level atomic oxygen flux was first established at an upstream $\mathrm{O}_{2}$ pressure of 100 Torr. The $\mathrm{O}_{2}$ was then pumped out, and the atomic oxygen signal began to decay. After an arbitrary interval of about 16 $h$, the 100 Torr of $\mathrm{O}_{2}$ was reapplied to the upstream side, and the original level of atomic oxygen flux was recovered. This confirmed the source of the atomic oxygen and demonstrated the tandem behavior of the two basic mechanisms of oxygen transport and ESD emission. Adjustment of the upstream pressure of $\mathrm{O}_{2}$ is an optional method of controlling the atomic oxygen flux but is not nearly as quick or easy as adjusting the incident electron flux.

The ion energy distribution for ESD of oxygen from pure $\mathrm{Ag}$ and $\mathrm{Ag}-0.5 \mathrm{Zr}$ alloy is presented in Fig. 8. A discussion of the distribution and the effects of the $\mathrm{Zr}$ have been previously reported. ${ }^{9}$ An estimate of the neutral distribution was accomplished by varying the lens voltage in the QMS from 0 $\mathrm{V}$ (where the background gases are repelled) to $+10 \mathrm{~V}$ where the most energetic oxygen neutrals are repelled. It was found that the mean energy for the neutrals is greater than $2 \mathrm{eV}$. Precise, neutral measurements are extremely difficult and have not yet been completed. Feulner has conducted experiments that have yielded neutral energy distributions with mean energies of less than $1 \mathrm{eV}$, but this was for much less

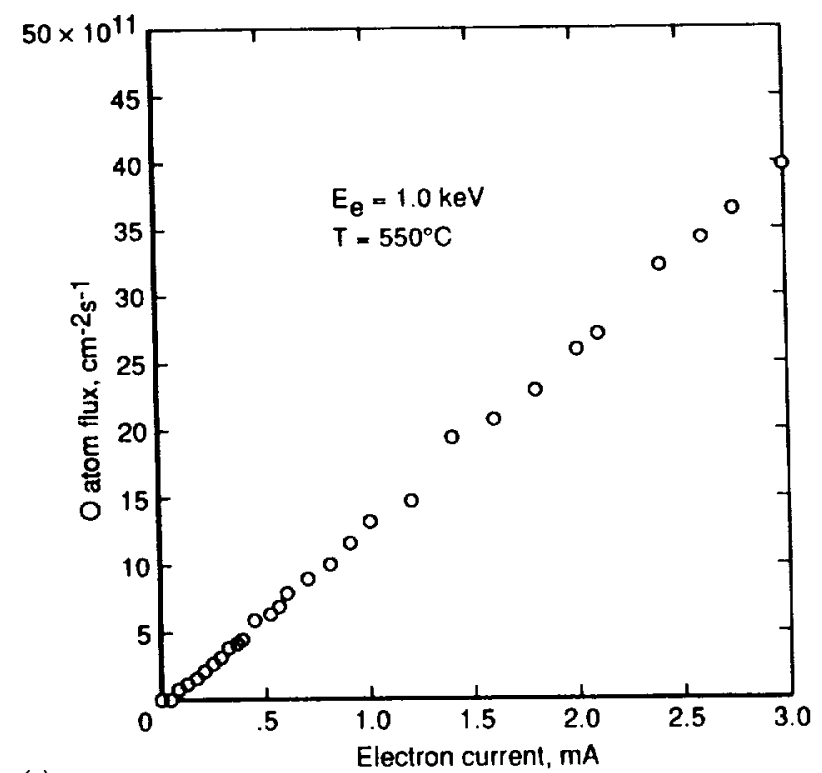

(a)

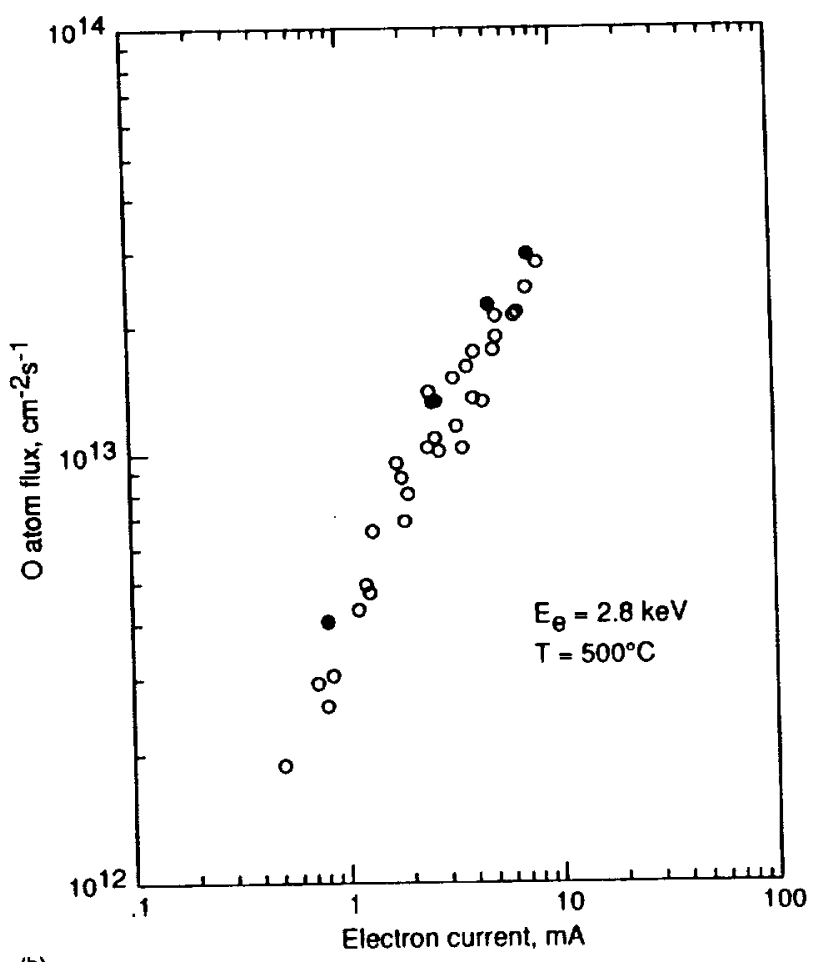

(b)

FIG. 6. (a) A plot of atomic-oxygen flux (pulse counting) versus electron bombardment current at $1 \mathrm{keV}$ energy and a membrane temperature of $550^{\circ} \mathrm{C}$. The $\mathrm{O}$ atom flux linearly increases with electron bombardment current, thus indicating the instrument is ESD limited. (b) A plot of atomicoxygen flux (analog) versus electron bombardment current at $2.8 \mathrm{keV}$ energy and a membrane temperature of $500^{\circ} \mathrm{C}$. The instrument is still ESI limited.

electron energy. ${ }^{10}$ This area of research is a most fertile and interesting ground for further study.

It is also important to characterize the internal energy of the oxygen atom beam, since it was designed to simulate the ground state flux that exists in low-Earth orbit. Figure 9(a) shows an appearance potential curve to examine the internal state of the emitted neutrals by the steps in the appearance 


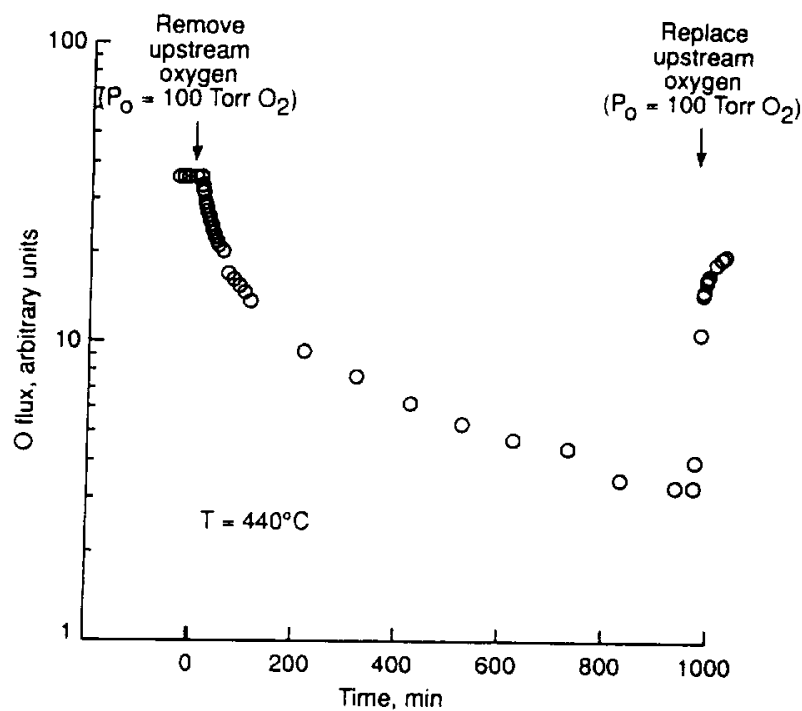

FIG. 7. The removal of the $\mathrm{O}_{2}$ upstream eliminates the source of oxygen atoms and ultimately the atomic-oxygen flux. At a membrane temperature of $440^{\circ} \mathrm{C}$, the atomic oxygen flux required over $16 \mathrm{~h}$ to decay a factor of 10 .

potential curves near the ionization energy for atomic oxygen. If there are excited states, e.g., ' $D$, then ionization should occur at a lower energy than the 13.6-13.7 eV necessary for the first ionization potential. This does not appear to be the case, since the only step observed is at $19.8 \mathrm{eV}$. When this measurement is corrected for the $W$ filament work function, which is $4.5 \mathrm{eV}$, and the effect of field leakage, which was determined to be $1.6 \mathrm{eV}$, then the predicted value is closely approached. In any case, the resulting electron kinetic energy is several $\mathrm{eV}$ higher than what would be expected for any excited state population. It is therefore probable that the ${ }^{3} \mathrm{P}$ ground state is the only state. The field leakage for the specific instrument geometry employed was estimated by using the Simion computer simulation. [See Fig. 9(b).]

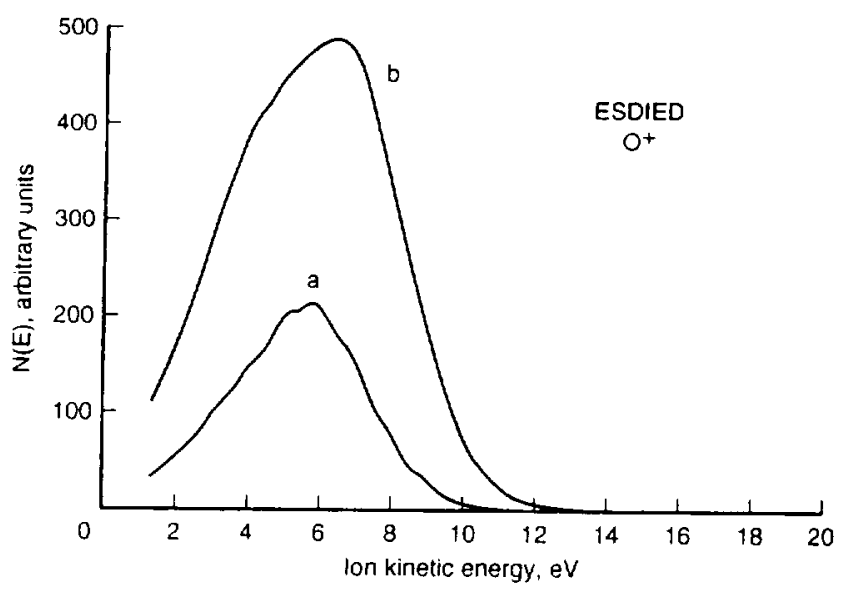

FiG. 8. The ion energy distribution for (a) pure Ag membrane which has a FWHM of approximately $4 \mathrm{eV}$ compared to (b) a $\mathrm{Ag}-0.5 \mathrm{Zr}$ alloy with a FWHM of $6 \mathrm{eV}$.

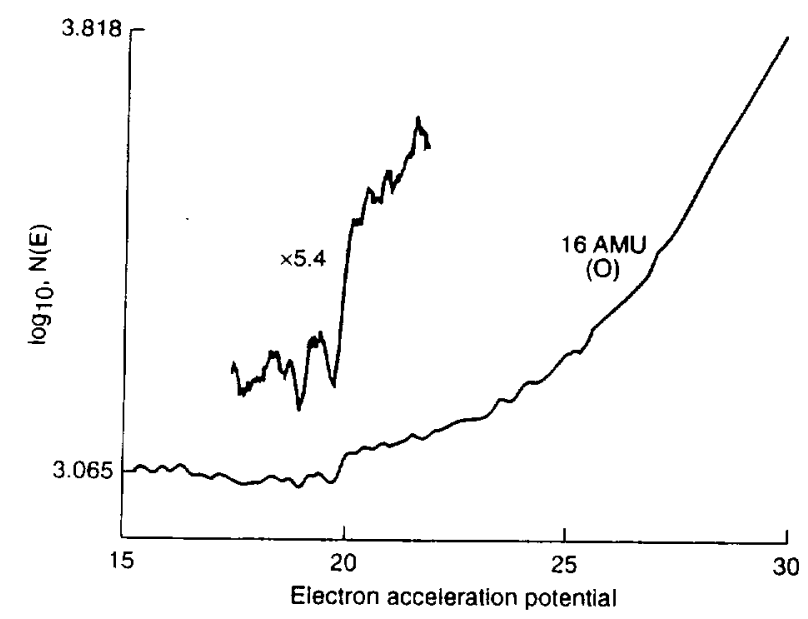

(a)

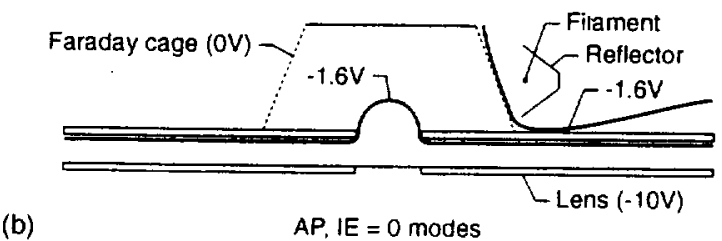

FIG. 9. A plot of the $\log N(E)$ vs QMS electron impact potential. The first ionization state corrected for the filament work function gives $E_{k}=15.3 \mathrm{eV}$. (b) A Simion computer simulation of field leakage into the ionizing region of the QMS gives a further correction of $1.6 \mathrm{eV}$ so that $E_{k}=13.7 \mathrm{eV}$, which is the measured first ionization potential for atomic oxygen. The internal energy was found to be the ${ }^{3} P$ state (the ground state) based on the single step in the $O$ signal $(m / e=16)$ at the appearance potential.

It appears quite likely that $1 \times 10^{14} \mathrm{~cm}^{-2} \mathrm{~s}^{-1}$ is achievable before reaching a transport or diffusion limited condition. The crossover point from ESD control to diffusion control can be easily estimated. Figure 10 represents the sequence of the initial steady state atomic oxygen flux, followed by the removal of the incident electron beam, and then the beam turned back on 5 min later. No change in the magnitude of the flux was observed, which indicates that the surface coverage was not changed, and, therefore, that the HOAG was ESD limited. This is consistent with the data in Fig. 6. The transport of oxygen through the membrane $\left(J_{\mathrm{O} / \mathrm{Ag}}\right)$ at steady state is given by

$$
J_{\mathrm{O} / \mathrm{Ag}}=\frac{2 K P_{0}^{1 / 2}}{d}=\frac{2 K_{0} P_{0}^{1 / 2}}{d} \exp \left(\frac{-E_{K}}{R T}\right)
$$

where $K$ is the permeability $\left(\mathrm{cm}^{-1} \mathrm{~s}^{-1}\right), P_{0}$ is the normalized upstream $\mathrm{O}_{2}$ pressure (atmospheric fraction), $d$ is the membrane thickness $(\mathrm{cm}), E_{K}$ is the activation energy for oxygen transport $\left(22.86 \mathrm{kcal} / \mathrm{mol}^{-1}\right), T$ is the membrane temperature $(\mathrm{K})$; and $K_{0}$ is the permeability preexponential $\left(5.2 \times 10^{18} \mathrm{~cm}^{-1} \mathrm{~s}^{-1}\right)$. The flux of ESD emitted neutrals and ions at steady state $\left(J_{0 / \mathrm{ESD}}\right)$ is given by

$$
J_{\mathrm{O} / \mathrm{Ag}}=Q \theta \sigma_{0} J_{e^{-}}
$$






FIG. 10. A plot of QMS signal as a function of time, with a 5-min absence of the electron bombardment. This suggests that the surface population of atoms has not significantly changed which, in turn, suggests an ESD limitation.

where $Q$ is the ESD cross section for neutral $\mathrm{O} / \mathrm{Ag}\left(7 \times 10^{-19}\right.$ $\left.\mathrm{cm}^{2}\right), \theta \sigma_{0}$ is the oxygen concentration $\left(\mathrm{cm}^{-2}\right)$, and $J_{e^{-}}$is the incident electron flux $\left(\mathrm{cm}^{-2} \mathrm{~s}^{-1}\right)$. At steady state when $d \theta / d t=0$, then

$$
J_{\mathrm{O} / \mathrm{Ag}}=J_{\mathrm{O} / \mathrm{ESD}}
$$

and the temperature where this occurs is given by

$$
T_{c}=\frac{E_{K}}{R}\left[\ln \left(\frac{2 K_{0} P_{0}^{1 / 2}}{Q \theta \sigma_{0} J_{e}^{-d}}\right)\right]^{-1} .
$$

At $5 \mathrm{~mA}$ incident $e^{-}$flux, 760 Torr upstream pressure, and a membrane thickness of $0.2 \mathrm{~mm}$ for example, a value of $T=412{ }^{\circ} \mathrm{C}$ is determined for the crossover temperature. It is desirable to reduce the membrane thickness to as thin as possible in order to produce sufficient oxygen transport at as low a membrane temperature as is possible. The theoretical limit for $J_{\mathrm{O} / \mathrm{Ag}}$ is controlled by the oxygen source, which is the solid-state transport of atoms through the Ag membrane, given by Eq. (3). Although we have employed 100 Torr upstream pressure for most measurements, the highest pressure utilized was 760 Torr $\mathrm{O}_{2}$. The thicknesses of the majority of membranes used in this work were between 0.25 and 0.38 $\mathrm{mm}$, but we have also fabricated rolled pinhole-free $12-\mu \mathrm{m}$ membranes supported by $\mathrm{Ag}$ mesh to withstand the differential pressure. Using values of the aforementioned parameters that maximize the flux, the highest possible, solid-state transport is $4 \times 10^{15} \mathrm{~cm}^{-2} \mathrm{~s}^{-1}$ and represents a theoretical limit for this process. The actual limitation observed in this work. however, is the ESD which controls the oxygen-atom emis- sion. Ultimately, the electron bombardment (which gives a proportional oxygen-atom emission) that can be applied is limited by the heating of the membrane. The temperature cannot exceed $550^{\circ} \mathrm{C}$ because of the observed on set of molecular desorption. Utilizing the previous parameters in Eq. (6), a crossover temperature of $314^{\circ} \mathrm{C}$ is determined for a supported $12-\mu \mathrm{m}$ thick membrane.

The actual emission-distribution function of the top hat membrane has not yet been determined. What has been reported in this work is that which has been observed at the QMS detector located $10 \mathrm{~cm}$ away. Oxygen-atom flux emitted at the membrane can be roughly estimated from Eq. (4) with previously measured cross-section data $\left(7 \times 10^{-16}\right.$ $\left.\mathrm{cm}^{2}\right),{ }^{7}$ the saturated Ag surface $(\theta \sim 0.5),{ }^{11}$ and an electron bombardment current of $10 \mathrm{~mA}$ (which for a surface area of $0.456 \mathrm{~cm}^{2}$ gives $J_{e^{-}}=1.37 \times 10^{17} \mathrm{~cm}^{-2} \mathrm{~s}^{-1}$ ). This generates an emitted oxygen-atom flux of approximately $1.2 \times 10^{14}$ $\mathrm{cm}^{-2} \mathrm{~s}^{-1}$, which is, as expected, greater than that indicated in Fig. 6(b) $\left(\sim 4.5 \times 10^{13} \mathrm{~cm}^{-12} \mathrm{~s}^{-1}\right)$ measured at the detector. Although this is not a representation of the flux distribution, it is indicative, however, of a predominantly directed flux, which may not be so surprising considering past observations of ESD species coming off along the bond direction. ${ }^{12}$

\section{A calibration experiment}

Recently, we have utilized the HOAG in an experiment to measure the accumulation of adsorbed atomic oxygen on the surface of polycrystalline $\mathrm{Au}$ in order to establish a measure of the incident flux. ${ }^{13}$ The Au was selected because it is well known that $\mathrm{O}_{2}$ will not adsorb, but atomic oxygen will. ${ }^{14}$ Further, theoretical and experimental determinations have indicated that oxygen atoms do not dissolve into $\mathrm{Au} .{ }^{15}$ The electron source used for ESD was a separate gun (ordinarily used for glancing incidence Auger electron spectroscopy) operated at a maximum electron density of $10 \mu \mathrm{A} \mathrm{cm}^{-2}$. This very low electron beam generated a corresponding low oxygen flux detected at the QMS. Ion scattering spectroscopy (ISS) was used to detect the accumulating atomic oxygen, since it is sensitive to only the surface layer and not to the underlying layers. The ISS was performed with a defocused, $1-\mathrm{keV} \mathrm{He}^{+}\left(100 \mathrm{nA}\right.$ over $\left.1 \mathrm{~cm}^{2}\right)$ beam, and detection was performed using the double pass cylindrical mirror analyzer operated in the nonretarded mode. No beam effects were observed, since the signal remained constant over repeated scans. The oxygen surface coverage on the $\mathrm{Au}$ was determined to be approximately 0.7 , which indicated the flux was $\sim 1.3 \times 10^{10} \mathrm{~cm}^{-2} \mathrm{~s}^{-1}$. This value was found to be consistent with QMS and ESD flux approximations. Since this experiment was conducted, the HOAG has been modified to include the integral circular filament. This has substantially increased the available electron flux and therefore the emilted flux [see Fig. 6(b)]. All data, other than this experimen:, were taken using the modified instrument.

\section{SUMMARY}

The development of a high purity, hyperthermal, continious beam atomic oxygen source capable of retrofitting to existing UHV systems has been achieved. The instrument 
complements a general system capability, while its small size and simplicity of design permits tailoring the instrument for most experimental geometries. The flux level has been measured to be $4.5 \times 10^{13} \mathrm{~cm}^{2} \mathrm{~s}^{-1}$ at $10 \mathrm{~cm}$ from the $\mathrm{Ag}$ emission plane and estimated to be $1.2 \times 10^{14} \mathrm{~cm}^{-2} \mathrm{~s}^{-1}$ at the emission plane. The emitted neutrals are ground state $\left({ }^{3} P\right)$, and the mean ion kinetic energy is $-5 \mathrm{eV}$ with a full width at half maximum (FWHM) of $4 \mathrm{eV}$. The energy of the oxygen atoms was found to be $>2 \mathrm{eV}$ and may be substantially reduced for other applications by collision with a temperature controlled, nonreactive surface (with a concomitant spread in the energy distribution).

\section{ACKNOWLEDGMENTS}

The authors gratefully acknowledge the comments of Professor Gar B. Hoflund of the University of Florida and the authors of Simion software which greatly enhanced the analysis.
'S. L. Koontz, K. Albyn, and L. J. Leger, J. Spacecraft 28, 315 (1991).

${ }^{2}$ L. J. Leger, I. K. Spiker, J. F. Kuminecz, T. J. Ballentine, and J. T Visentine, AIAA Paper 8-2631, November 1983

${ }^{3}$ L. J. Leger, J. T. Visentine, and J. F. Kunminecz, AlAA Paper 84-0548, January 1984.

${ }^{4} \mathrm{~J}$. B. Cross, in Proceedings of the Workshop on Surface Reactions in the Space Environment, Northwestern University, 1989.

${ }^{5} \mathrm{~A}$. Chutjian, O. J. Orient, and E. Murad, in Ref. 4.

${ }^{6} \mathrm{~J}$. T. Visentine, NASA Tech. Memor. No. 100459 (1988).

${ }^{7}$ R. A. Outlaw, Gar B. Hoflund, and G. R. Corallo, Appl. Surf. Sci. 28, 235 (1987).

${ }^{8}$ G. B. Hoflund, M. R. Davidson, and R. A. Outlaw, Surf. Interface Anal. 19, 325 (1992).

${ }^{9}$ Mark R. Davidson, Gar B. Hoflund, and R. A. Outlaw, Surf. Sci. 281, 111 (1993).

${ }^{10} \mathrm{P}$. Feulner, Desorption Induced by Electronic Transitions, DIET II, Proceedings of the 2 nd International Workshop, edited by W. Brenig and D. Menzel (Springer, Heidelberg, 1985), p. 142.

${ }^{11} H$. A. Engelhardt and D. Menzel, Surf. Sci. 57 (1976) 591.

${ }^{12}$ T. E. Madey and J. T. Yates, Jr., Surf. Sci. 63 (1977) 2093

${ }^{13}$ M. R. Davidson, Gar B. Hoflund, and R. A. Outlaw, J. Vac. Sci. Technol. A 11, 1 (1993)

${ }^{14}$ N. D. S. Canning, D. Outka, and R. J. Madix, Surf. Sci. 141, 240 (1984),

${ }^{15}$ M. E. Eberhart, M. M. Donovan, and R. A. Outlaw, Phys. Rev. B 46 , 12744 (1992). 
